\title{
Über die Thalliumlegierungen.
}

\author{
Von \\ N. S. Kurnakow und N. A. Puschin. ${ }^{1}$ \\ Mit 3 Figuren im Text.
}

Die früheren Untersuchungen ${ }^{2}$ zeigten, dafs die Alkalimetalle mit Quecksilber, Blei und Wismut eine ganze Reihe von bestimmten Verbindungen bilden, die durch die Temperaturmaxima in den Schmelzpunktskurven aulserordentlich scharf charakterisiert sind.

Man konnte nun erwarten, dafs das Thallium, welches in der. elften (ungeraden) Reihe des periodischen Systems, zwischen Quecksilber und Blei, steht, analoge Verhältnisse zeigen würde und die Resultate dieser Untersuchung bestätigen diese Annahme vollständig. Anfangs beabsichtigten wir nur die Legierungen des Thalliums mit Kalium und Natrium zu studieren, aber die dabei gemachten Beobachtungen zwangen uns, auch einige Schwermetalle saurer Natur und zwar: Quecksilber, Zinn, Cadmium, Blei, Wismut und Antimon in den Kreis unserer Untersuchung zu ziehen. Bekanntlich zeigt das Thallium in den salzartigen Verbindungen einen zwiespältigen Charakter; es verhält sich in den Oxydulsalzen (TIX) wie ein Alkalimetall; in den Oxydsalzen $\left(\mathrm{TIX}_{3}\right.$ ) dagegen fungiert es als dreiwertiges Schwermetall. Ein ähnlicher Unterschied in den chemischen Funktionen, wenn auch in etwas anderer Form, ist bei den metallischen Legierungen des Thalliums zu beobachten.

In den Verbindungen mit Kalium, Natrium, sowie mit Zinn und Cadmium verbält es sich äbnlich den Schwermetallen saurer Natur, wie dem Quecksilber und besonders dem Blei, denen es ja auch im freien Zustande den physikalischen Eigenschaften nach sehr nahe steht. Andererseits mufs man annehmen, dafs es in den Legierungen

${ }^{1}$ Ins Deutsche übertragen von M. Koss in Berlin.

${ }^{2}$ N. Kunnakow, Journ. russ. chem. Ges. 31, 929; Zeitschr. anorg. Chem. 93, 439 . 
mit $\mathrm{Hg}, \mathrm{Pb}, \mathrm{Bi}$ die Funktion eines Alkalimetalls hat. So schmilzt zum Beispiel die Verbindung $\mathrm{TlHg}_{2}$ obne Zersetzung; sie entspricht in ihrer Zusammensetzung den früher beschriebenen beständigen Alkalianalgamen $\mathrm{NaHg}_{2}$ und $\mathrm{KHg}_{2}$.

Die Untersuchung der Legierungen des Thalliums mit Blei und Wismut ist vorläufig noch nicht vollständig abgeschlossen, da sich dabei zum ersten Mal charakteristische neue Eigentümlichkeiten in den Schmelzpunktskurven zeigten, die noch ein eingehendes Studium verlangen.

Die vorliegende Abhandlung enthält die Resultate, die bei der Untersuchung der Schmelztemperaturen der Thalliumlegierungen: 1) mit Natrium und Kalium und 2) mit Zinn, Cadmium und Quecksilber ${ }^{1}$ erhalten worden sind.

\section{Die Legierungen des Thalliums mit Natrium und Kalium.}

Die zur Bestimmung der Schmelzbarkeit der Doppelsysteme $\mathrm{Na}+\mathrm{Tl}$ und $\mathrm{K}+\mathrm{Tl}$ angewendete Untersuchungsmethode unterscheidet sich dem Wesen nach wenig von der früher bei den Alkaliamalgamen beschriebenen. $^{2}$ Das metallische Kalium und Natrium wurden sorgfältig von der Oberflächenschicht befreit, die sich in dem zur Aufbewahrung verwendeten Vaselinöl angesetzt hatte. Das anhaftende Öl wurde zuerst durch Behandlung mit Benzin, nachher mit frisch destilliertem und über metallischem Natrium aufbewahrtem Äther

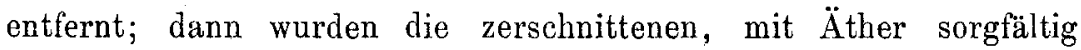
gewaschenen Metallstücke in kurzen, weiten, fest verschlossenen Probiergläsern gewogen und schnell in eiserne Tiegel geworfen, in denen sich flüssiges Vaselin oder geschmolzenes Paraffin befand, um die Oxydation des Metalls zu verhindern. Bei den Legierungen des Kaliums mit kleinen Mengen von Thallium diente ein Gemisch von Vaselinöl mit Benzin als schützende Schicht. Zur bestimmten Gewichtsmenge des Alkalimetalls wurde allmählich die vorher abgewogene Menge metallischen Thalliums (von MERK in Darmstadt) zugegeben und die Temperaturen bei Beginn der Krystallausscheidung beobachtet. Die Schmelze wurde fortwährend gemischt. Wenn es möglich war, wurde auch der Thermometerstand bei der Abkühlung

1 Siehe unsere Mitteilungen in den Sitzungen der Abteilung der Chemie der russisch. Physik:-Chem. Gesellsch. am 14. Sept. und 7. Dez. 1900. (Journ. russ. chem. Ges. 32, 633. 830.)

${ }^{2}$ Journ russ. chem. Ges. 31, 929; Z. anorg. Chem. 23, 441. 
der fest gewordenen Masse in den Übergangs- und eutektischen Punkten ermittelt. Nach einer Reihe von Bestimmungen wurde die erkaltete Legierung mit Benzin und Äther gewaschen und gewogen; dabei betrug gewöhnlich die Differenz der gefundenen und berechneten Menge nicht mehr als $0.1-0.2 \mathrm{~g}$ bei einem Gesamtgewicht von 100 oder mehr als 100 Gramm. Ein Teil der auf diese Weise gewonnenen Legierung, deren Thalliumgehalt bekannt war, diente wiederum zur Gewinnung von Legierungen mit sehr hohem Thalliumgebalte. Zn diesem Zwecke wurden aus den wieder unter einer Paraffinschicht geschmolzenen Legierungen mittels eines eisernen Löffels einzelne Portionen herausgeschöpft und in Vaselinöl abgekühlt; sodann wurden gehörig ausgewaschene Kügelchen derselben gewogen und $\mathrm{zu}$ einer bestimmten Thalliummenge hinzugefügt. Merkwürdigerweise wird die Auflösung einer Kalium-Thalliumlegierung in überschüssigem Thallium von starkem Zischen und Erwärmung der metallischen Masse begleitet, was auf eine grolse Wärmeentwickelung bei der Einwirkung der Komponenten hindeutet. Die Energie der bezeichneten Reaktion vermindert sich bei der Annäherung an den eutektischen Punkt (15.7 Atomprozente K). Der erhebliche Wärmeeffekt bei der Bildung deutet auf eine Verwandtschaft der Kalium-Thalliumlegierungen mit den Alkaliamalgamen. Um bei der Abkühlung den Wärmeverlust geringer zu machen. befand sich der Tiegel auf einer Asbestunterlage in einem unten geschlossenen Thoncylinder; der Boden desselben hatte in der Mitte eine runde Öffnung, in welche ein Brenner eingesetzt werden konnte. Nach dem Schmelzen des Metalles im Tiegel wurde der Brenner entfernt, und die Öffnung mit einem Asbeststopfen zur Verhinderung der Zirkulation der im Cylinder befindlichen erwärmten Luft geschlossen. Der obere T'eil des Tiegels wurde mit Asbest bedeckt, in dem sich zwei Löcher für das Thermometer und den Rührer befanden. Die erhaltenen Resultate sind in den Tabellen I und II zusammengestellt. In der ersten Spalte sind die Nummern der Versuche verzeichnet, in der zweiten bezw. dritten der Gehalt der untersuchten Legierungen an Alkalimetall ( $\mathrm{Na}, \mathrm{K}$ ) bezw. an Thallium in Atomprozenten, in der vierten sind die entsprechenden Schmelztemperaturen (Anfang der Ausscheidung von Krystallen) aufgeführt, in der fünften endlich ist der Thermometerstand notiert, wo bei weiterer Abkühlung der metallischen Masse Übergangs- oder eutektische Punkte auftraten. Dieselben sind nur dort angegeben, wo die eutektischen Punkte mit unzweifelhafter Deutlichkeit beobachtet werden konnten. 
Tabelle I.

Legierungen des Thalliums mit Natrium.

\begin{tabular}{|c|c|c|c|c|c|}
\hline \multirow{2}{*}{$\begin{array}{c}\dot{\Phi} \\
\stackrel{g}{\Xi} \\
\stackrel{B}{Z}\end{array}$} & \multicolumn{2}{|c|}{ Atomprozente } & \multirow{2}{*}{ 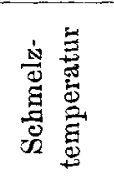 } & \multirow{2}{*}{ 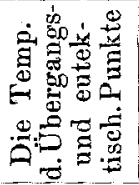 } & \multirow{2}{*}{ Bemerkungen } \\
\hline & $\mathrm{Na}$ & TI & & & \\
\hline 1 & 100.00 & 0.00 & $97.0^{\circ}$ & - & \multirow{11}{*}{$\begin{array}{l}\text { Atomerniedrigung }=4.28^{\circ} . \\
\text { Atomemiedrigung }=431^{\circ} .\end{array}$} \\
\hline 2 & 99.65 & 0.35 & 95.5 & - & \\
\hline 3 & 99.20 & 0.80 & 93.4 & - & \\
\hline 4 & 98.66 & 1.34 & 91.3 & - & \\
\hline 5 & 98.09 & 1.91 & 88.9 & - & \\
\hline 6 & 97.22 & 2.78 & 84.6 & 一 & \\
\hline 7 & 96.43 & 3.57 & 81.1 & - & \\
\hline 8 & 95.86 & 4.14 & 78.6 & - & \\
\hline 9 & 95.21 & 4.79 & 75.1 & - & \\
\hline 10 & 94.54 & 5.46 & 72.0 & - & \\
\hline 11 & 93.95 & 6.05 & 68.7 & - & \\
\hline 12 & 92.80 & 7.20 & 64.1 & $64.1^{\circ}$ & Eutektischer Punkt $A$. \\
\hline 13. & 91.92 & 8.08 & - & 64.1 & \multirow{7}{*}{ Harte oktaëdrische Krystalle. } \\
\hline 14 & 91.34 & 8.66 & - & - & \\
\hline 15 & 90.93 & 9.07 & - & - & \\
\hline 16 & 90.14 & 9.86 & - & 一 & \\
\hline 17 & 89.27 & 10.73 & 73.8 & 64.0 & \\
\hline 18 & 88.18 & 11.82 & 75.4 & - & \\
\hline 19 & 87.01 & 12.99 & 77.0 & - & \\
\hline 20 & 85.93 & 14.07 & 77.9 & 77.9 & Übergangspunkt $B$. \\
\hline 21 & 81.88 & 18.12 & ca. 108 & - & \multirow{13}{*}{ Lange Prismen. } \\
\hline 22 & 80.22 & 19.78 & 120.2 & - & \\
\hline 23 & 78.20 & 21.80 & 128.6 & - & \\
\hline 24 & 77.39 & 22.61 & 133.2 & - & \\
\hline 25 & 76.90 & 23.10 & 135.3 & - & \\
\hline 26 & 76.24 & 23.76 & 138.5 & - & \\
\hline 27 & 75.40 & 24.60 & 142.6 & - & \\
\hline 28 & 74.70 & 25.30 & 145.2 & - & \\
\hline 29 & 74.00 & 26.00 & 148.4 & - & \\
\hline .30 & 72.45 & 27.55 & 153.0 & - & \\
\hline 31 & 71.88 & 28.12 & 155.5 & - & \\
\hline 32 & 71.23 & 28.77 & 156.7 & - & \\
\hline 33 & 70.70 & 29.30 & 158.2 & $=$ & \\
\hline 34 & 70.10 & 29.90 & 158.7 & 158.7 & Übergangspunkt $C$. \\
\hline
\end{tabular}


Tabelle I (Fortsetzung).

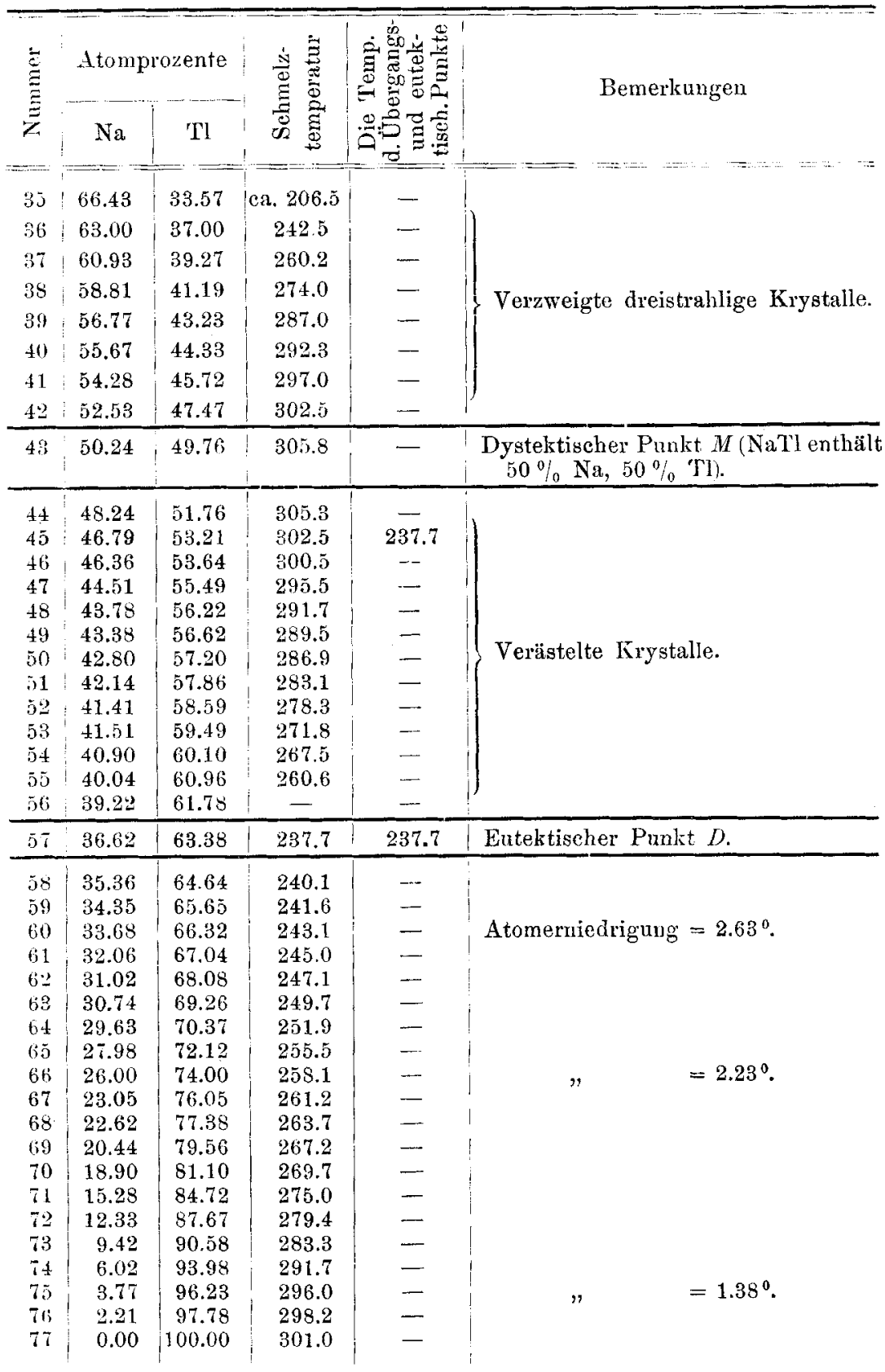


T'abelle II.

Legierungen des Thalliums mit Kalium.

\begin{tabular}{|c|c|c|c|c|c|}
\hline \multirow{2}{*}{ 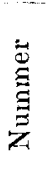 } & \multicolumn{2}{|c|}{ Atomprozente } & \multirow{2}{*}{ 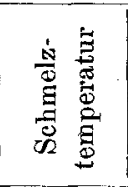 } & \multirow{2}{*}{ 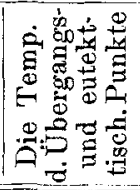 } & \multirow{2}{*}{ Bemerkungen } \\
\hline & $\mathrm{K}$ & $\mathrm{Tl}$ & & & \\
\hline 1 & 100.00 & 0.00 & $\equiv 62.5^{\circ}$ & $\ldots$ & \\
\hline 2 & 97.67 & 2.33 & 62.5 & $65.2^{\circ}$ & \\
\hline 3 & 96.70 & 3.30 & ca. 161.0 & - & \\
\hline 4 & 94.76 & 5.24 & 172.5 & - & \\
\hline 5 & 91.56 & 8.44 & ca. 192.5 & - & \\
\hline 6 & 88.67 & 11.33 & ca. 206 & - & \\
\hline 7 & 86.98 & 13.02 & ca. 211.2 & - & \\
\hline 8 & 84.26 & 15.74 & 217.0 & - & \\
\hline 9 & 82.51 & 17.49 & 220.5 & 62.5 & \\
\hline 10 & 78.31 & 21.69 & 227.3 & - & \\
\hline 11 & 76.84 & 23.16 & 229.3 & - & \\
\hline 12 & 74.32 & 25.68 & 232.5 & 62.5 & \\
\hline 13 & 70.63 & 29.67 & 237.7 & - & \\
\hline 14 & 68.63 & 31.37 & 240.0 & - & \\
\hline 15 & 67.06 & 32.94 & 242.0 & - & Übergangspunkt $E$. \\
\hline 16 & 62.06 & 37.94 & 254.2 & - & \\
\hline 17 & 58.09 & 41.91 & 270.5 & - & \\
\hline 18 & 54.17 & 45.83 & 309.0 & - & \\
\hline 19 & 52.04 & 47.96 & 326.7 & - & \\
\hline 20 & 49.98 & 50.02 & 335.0 & - & Dystektischer Punkt $N(\mathrm{KTl})$, \\
\hline 21 & 47.47 & 52.53 & 331.0 & - & \\
\hline 22 & 43.87 & 56.13 & 321.5 & - & \\
\hline 23 & 38.00 & 62.00 & 313.2 & - & \\
\hline 24 & 31.82 & 68.18 & 290.0 & - & \\
\hline 25 & 28.16 & 71.84 & 280.0 & - & Harte kubische Krystalle. \\
\hline 26 & 24.07 & 75.93 & 248.2 & - & \\
\hline 27 & 21.13 & 78.87 & 222.0 & 172.5 & \\
\hline 28 & 18.08 & 81.92 & 195.5 & - & \\
\hline 29 & 15.70 & 84.30 & 172.5 & 172.5 & Eutektischer Punkt $F$. \\
\hline 30 & 13.38 & 86.62 & 195.0 & - & \\
\hline 31 & 9.83 & 90.17 & 234.0 & - & Atomerniedrigung $=9.14^{\circ}$. \\
\hline 32 & 6.56 & 93.44 & 264.7 & - & $=7.73^{\circ}$. \\
\hline 33 & 4.16 & 95.84 & 288.5 & - & $=5.93^{\circ}=$ \\
\hline 34 & 1.85 & 98.15 & 297.1 & - & $=2.88^{\circ}$ \\
\hline 35 & 0.00 & 100.00 & 301.0 & - & $=2.12^{\circ}$ \\
\hline
\end{tabular}


Wenn wir die Verhältnisse der Atomprozente auf der Abscissenaxe und die entsprechenden Schmelztemperaturen und die der Übergangspunkte auf der Ordinatenaxe auftragen, so bekommen wir Diagramme der Schmelzbarkeit, wie sie die Figur 1 darstellt. Die Zeichnung zeigt deutlich, dals die Legierungen des Thalliums mit Kalium und Natrium dieselben bemerkenswerten Eigenschaften aufweisen, die bei den Alkaliamalgamen früher festgestellt wurden. Hier wie dort bestehen die Schmelzpunktkurven aus einer Anzahl von Ästen, die bestimmten chemischen Verbindungen angehören; einige derselben charakterisieren sich durch sehr hohe Temperaturmaxima, die oberhalb der Schmelzpunkte der einzelnen das betreffende Doppelsystem bildenden Komponenten liegen. Dies Verhalten kommt bekanntlich den stabilen Repräsentanten chemischer Verbindungen zu, z. B. den Verbindungen der Metalle mit den Metalloiden u. s. w., bei denen der Bildungsvorgang tiefgehende Änderungen in den Eigenschaften der reagierenden Körper hervorbringt. Für die Amalgame des Kaliums und Natriums entsprechen solche schwer schmelzbaren oder ,dystektischen" "Systeme bestimmten Verbindungen vom Typus: $\mathrm{RHg}_{2}(\mathrm{R}=\mathrm{K}, \mathrm{Na})$. Bei den entsprechenden Legierungen des Thalliums gehören die dysteltischen Punkte $M$ und $N$ (Figur 1) den Verbindungen der einfachen Zusammensetzung: $R T l(R=\mathrm{Na}$, K) an.

Die Substanzen NaTl und KTl schmelzen bei $305.8^{\circ}$ und $335.0^{\circ}$, d. h. bedeutend höher als das Thallium (Schmelzpunkt $301.7^{\circ}$ ), der am schwersten schmelzbare Bestandteil des Systems. Betrachten wir zunächst die untersuchten Legierungen des Natriums mit Thallium genauer. Hier hat die Schmelzpunktskurve Na $A B C M D \mathrm{Tl}$ vier charakteristische Punkte $A, B, C, M, D$, deren Lage durch folgende Temperaturen und Atomverhältnisse (Na: Tl) in der flüssigen Phase bestimmt wird: (Fig. 1, S. 93.)

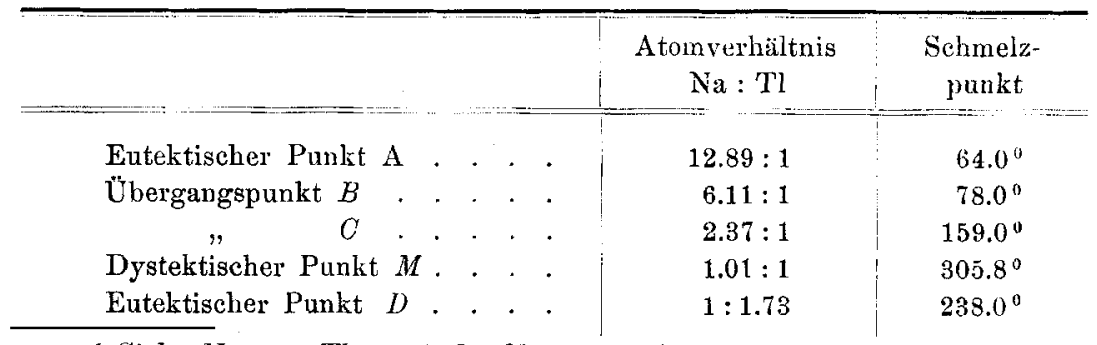

"Siehe Nennst, Theoretische Chemie, 2. Aufl., S. 126, „dystektische" oder schwerschmelzbare Substanzen kann man bis zum gewissen Grade den leichtschmolzbaren oder den "eutektischen" entgegenstellen. 
Die Punkte teilen die ganze Kurve in fünf verschiedene $A b$ schnitte $\mathrm{Na} A, A B, B C, C M D$ und $D \mathrm{Tl}$, die den selbständigen fünf festen Phasen entsprechen.

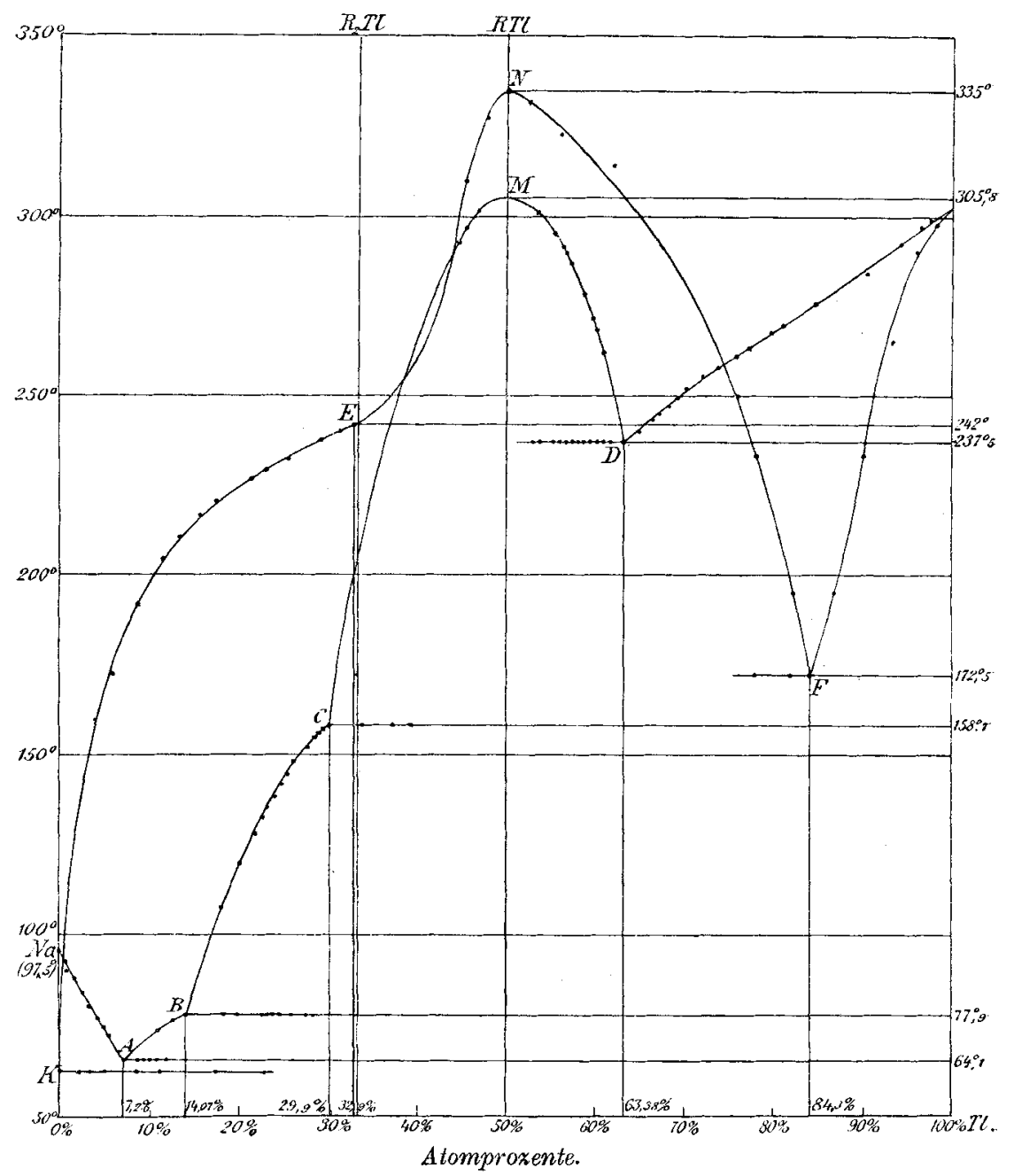

Fig. 1. Legierungen des Thalliums mit Natrium und Kalium.

Die an die eutektischen Punkte $A$ und $D$ sich anschliefsenden Kurvenzüge nach $\mathrm{Na}$ und $\mathrm{Tl}$ deuten die Ausscheidung des Natriums. und des Thalliums in freiem Zustande an. Sie gestalten sich etwa als gerade Linien, womit auch die sich verbältnismälsig wenig verändernde atomare Schmelzpunktserniedrigung innerhalb jener Kurven 
übereinstimmt. Für den Abschnitt $\mathrm{Na} A$ schwankt die Schmelzpunktsenniedrigung des Natriums, bei der Auflösung von einem Atome Thallium in 100 Atomen $\mathrm{Na}(100 \times 23 \mathrm{~g})$ zwischen 4.28 und 4.86. ${ }^{1}$ Diese Zablen nähern sich den von Hexcock und Neviuse bei den kryoskopischen Untersuchungen der verdünnten Lösungen des Thalliums in $\mathrm{Na}$ (bis 4.38 Atome $\mathrm{Tl}$ auf 100 Atome $\mathrm{Na})^{2}$ ermittelten Grölsen (3.9-4.7). Dieselben Werte der Depressionskonstante ergeben sich z. B. bei den Auflösungen von Gold in Natrium (5.4-4.5) oder in Quecksilber (4.4-4.6). Der Kurvenzug $D$ Tl wird charalsterisiert durch die aulserordentlich geringe atomare Schmelzpunktsdepression, die der Ausscheidung des Thalliums als feste Phase beim Zusammenschmelzen mit Natrium entspricht. Bei bedeutenden Veränderungen der Na-Konzentration (0-35 Atomproz.) ist die Atomdepression des Thalliums $=1.38-2.63,{ }^{3}$ was $1 / 4-1 / 2$ der normalen Verminderung 6.0-6.8 gleichkommt, die dem Thallium in freien Legierungen mit $\mathrm{Au}, \mathrm{Pt}, \mathrm{Ag}^{4}$ eigentümlich ist. Eine ebenso anormal geringe Depressionskonstante $(1.1-1.5)$ wurde von Heycock und Nevisue ${ }^{5}$ und ron A. N. KusnezofF ${ }^{6}$ bei den Lösungen des Natriums in Blei beobachtet. Mangels experimenteller Daten kann man jedoch heute noch nicht endgültig feststellen, ob diese anormale Depressionen von der Bildung fester Lösungen des Natriums in Blei und Thallium, oder von einer Komplexbildung der Atome des metallischen $\mathrm{Na}$ herrühren.

Die mittleren Abschnitte der Kurve $A B, B C$ und $C M D$ müssen drei verschiedenen chemischen Verbindungen angehören. Die Untersuchung der krystallisierten festen Körper steht in voller Übereinstimmung mit den Ergebnissen der Schmelzpunktsmethode. Der

${ }^{1}$ Die Berechnung der atomaren Depression $(K)$ nach den Angaben der Tabelle I erfolgte nach der Formel: $K=\frac{100 \Delta}{A B}$, wo $A$ die Emiedrigung der Schmelztemperatur der metallischen Lösung, $A$ und $B$ Atomprozentgehalt der beiden Metalle in der Legierung sind.

${ }^{2}$ Hexcocr u. Nevilue, Journ. Chem. Soc. 5 à (1889), 671.

- Die Veränderlichkeitsgrenzen für die Schmelzpunktserniedrigung rücken bedeutend näher zusammen, wenn wir die Erniedrigung, wie es auch die Konstruktion des Sehmelzpunlstscliagramms erfordert, nicht auf 100 Atome des lösenden Metalles, sondern auf 100 Atome beier Metalle, welche die Legierung bilden, beziehen.

${ }^{4}$ Heycock u. Nevilue, Journ. Chem. Soe. 1894, 34.

5 Journ. Chem. So.c. $1892,904$.

- Journ. russ. chern. Ges. 31 (1899), 944. 
Zweig $C M D$ stellt zweifellos ein Gebiet der stabilen Existenz einer bestimmten Verbindung NaTl dar, da das Atomverhältnis Na:Tl im dystektischen Punkt $M$ sich sehr dem Verhältnisse 1:1 nähert, und die beobachtete Differenz (0.24 Prozentatome Na) nicht aufserhalb der Fehlergrenzen des Versuches fällt. Es ist sehr wahrscheinlich, dafs die in dem Punkte $M$ beobachtete Zusammensetzung bei einer grölseren Anzahl von Versuchen über das Schmelztemperaturmaximum noch mehr mit dem nach dem Gesetze der multiplen Proportionen sich ergebenden Verhältnis übereinstimmen würde. Jedenfalls entspricht die Zusammensetzung im Punkte $N$ (Tabelle II) für das entsprechende Maximum der Kalium-Thalliumkurve ganz genau der definierten Verbindung KTl. Die Verbindung NaTl krystallisiert in dreistrahligen, baumförmigen Gebilden, deren Äste einen Winkel von $120^{\circ}$ bilden (rhomboëdrisches Krystallsystem). In der Nähe des Maximums $M$ treten diese baumförmigen Krystallisationen weniger deutlich hervor, und die mikroskopische Untersuchung ergiebt, dals die Legierung aus polygonalen kompakten, von einander durch Risse getrennten Körnern besteht. Wie bekannt, ist eine derartige Struktur gewöhnlicb den homogenen chemischen Substanzen eigen. Durch die Feuchtigkeit der Luft wird $\mathrm{NaTl}$ langsam angegriffen; dabei wird die zinnweifse Fläche der Substanz grau und zerfressen. Eine dem 'Typus nach analoge Verbindung $\mathrm{PbNa}$ ist auch von Jonnsis ${ }^{1}$ in Blei-Natriumlegierungen gefunden worden, die in ihren Eigenschaften in vielen Beziehungen den Thalliumverbindungen ähnlich sind. Der dem Kurvenzweige $B C$ entsprechende Körper $\mathrm{Na}_{\mathbf{x}} \mathrm{Tl}$ geht aus dem festen in den flüssigen Zustand unter Zersetzung über; das Schmelzdiagramm kann deshalb hier nieht die Zusammensetzung der festen Phase ebenso genau darstellen, wie es für den Abschnitt $C M D$ möglich war. Dennoch lärst auch in diesem Falle die Lage des Übergangspunktes die Grenzen erkennen, zwischen denen sich die chemische Verbindung von konstanter Zusammensetzung befinden mufs. ${ }^{2}$

Betrachten wir beispielsweise zwei Schmelzkurven $R P M$ und $O M L$, die sich in dem Übergangspunkte $M$ schneiden (Figur 2). Die erste Kurve hat das Maximum $P$, dessen Lage die Zusammensetzung der entsprechenden definierten Verbindung ermitteln und

1 JOANNIS, Compt. rend. 114 (1892), 585.

${ }^{2}$ N. Kurnakow, Journ. russ. chem. Ges. 31 (1899), 937. „Ermittelung bestimmter Verbindungen in den Legierungen nach der Schmelzmethode". Ber. K. R. T. Ges. 35 (1901), 7. 
sie durch die Formel $A_{n} B$ ausdrücken lälst: der Koëffizient $n$ stellt hier diejenige Zahl der Atome des Metalls A dar, die in der Verbindungen auf ein Atom des Metalls B kommt. Nehmen wir an, dafs die zweite Kurve $O M L$ einer anderen, durch die unbekannte Formel $A_{x} B$ ausgedrückten Verbindung entspricht, die unter Zersetzung in Übergangspunkte schmilzt und deren flüssige Phase die Zusammensetzung $A_{m} B$ hat. Der Wert des Koëffizienten in der letzten Formel wird unmittelbar bei dem Versuche bestimmt.

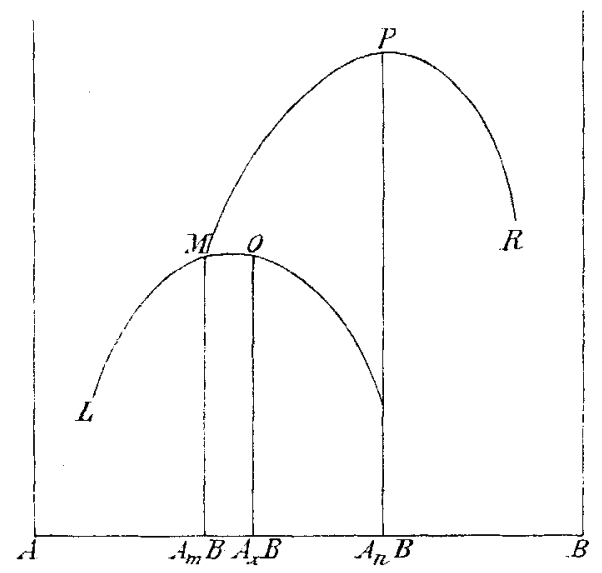

Fig. 2.

In allen bis jetzt erforschten Fällen von Doppelsystemen (Salzhydrate, Doppelsalze, Legierungen) lassen sich aufeinanderfolgende Änderungen in der Zusammensetzung der flüssigen Phasen und der aus ihnen sich auscheidenden Verbindungen beobachten. Der Unterschied liegt hauptsächlich darin, dafs in den flüssigen Lösungen die Veränderung der Verhältnisse der Bestandteile kontinuierlich geschieht, während die Zusammensetzung der entsprechenden festen Phasen, oder der chemischen Verbindungen sich - nach dem Gesetze der multiplen. Proportionen - sprungweise verändert. Bei kontinuierlicher Vergrörserung der Konzentrationen des einen, die flüssige Lösung bildenden Bestandteiles, beispielsweise von $A$, lärst sich der Wert des Koëffizienten $X$ in der allgemeinen Formel $A_{x} B$ für feste Phasen als eine allmählich steigende Reihe der einfachen Zahlen oder deren Verhältnisse darstellen. Aus diesem Grunde mufs auch der Koëffizient $X$ für zwei neben einander bestehende Doppelverbindungen $A_{n} B$ und $A_{x} \mathrm{~B}$, deren Zusammengehörigkeit in dem Übergangspunkte $M$ zu beobachten ist, gröfser als $n$ sein. Zugleich zeigt auch 
die Lage des Utbergangspunktes $M$, dafs die Gröfse $x$ kleiner als $m$ sein muis.

Wir haben also für den Zahlenwert von $x$ zwei Grenzen eine obere und eine untere, die sich aus der Ungleichung

ergeben.

$$
m>x>n
$$

Im allgemeinen wird die untere Grenze für $x$ durch die $\mathrm{Zu}$ sammensetzung derjenigen angrenzenden Verbindung gegeben sein, in welche sich die gesuchte Substanz $A_{x} B$ bei der Verwandlung im Übergangspunkte umwandelt, und die obere durch die Zusammensetzung der flüssigen Phase des Übergangspunktes. ${ }^{1}$

Wenn wir die Ungleichheit (1) auf die Verbindung $\mathrm{Na}_{\mathbf{x}}{ }^{\mathrm{T}} \mathrm{Tl}$ an-

1 Wenn die Formel der Verbindung $A_{n} B$ unbekannt ist, erhalten wir für die obere Grenze:

$$
x<m \text {. }
$$

Für viele stabilere Doppelkörper ist die Grölse der Differenz: $m-x$ dem Werte nach nicht sehr bedeutend, d. h. dafs der Übergangspunkt $M$ oft nahe an dem schwankenden Temperaturmaximum $O$ der gegebenen Substanz $A_{x} B$ liegt (Fig. 2). Als Bestätigung dessen dienen auch diejenigen flüssigen Phasen in den Übergangspunkten, die die höchsten Temperaturgrenzen einer stabilen Existenz für die Hydrate von $\mathrm{CaCl}_{2}$ und $\mathrm{MgCl}_{2}$, die unter Zersetzung schmelzen, darstellen.

Hydrate des Chlorealciums (Roozeвoom, Zeitschr. phys. Chem. 4, 33).

\begin{tabular}{c|c} 
Verhältnis & $\mathrm{CaCl}_{\frac{2}{2}}: \mathrm{H}_{2} \mathrm{O}$ \\
im Übergangspunkt & im festen Hydrat \\
$1: 2.07$ & $\begin{array}{c}1: 2 \\
1: 4.73\end{array}$ \\
$1: 4.83$ & $\begin{array}{c}1: 4\left(\alpha \mathrm{CaCl}_{2} .4 \mathrm{H}_{2} \mathrm{O}\right) \\
1: 4\left(\beta \mathrm{CaCl}_{2} .4 \mathrm{H}_{2} \mathrm{O}\right)\end{array}$
\end{tabular}

Hydrate des Chlormagnesiums (vay'x Hofe u. Meyerhofer, Zeitschr. phys. Chem. 27, 81).

\begin{tabular}{c|c}
\hline \multicolumn{2}{c}{ Verhältnis $\left(\mathrm{H}_{2} \mathrm{O}\right) \mathrm{MgCl}_{2}: \mathrm{H}_{2} \mathrm{O}$} \\
im Übergangspunkt & im festen Hydrat \\
\hline $1: 4.2$ & $1: 4$ \\
$1: 6.1$ & $1: 6$ \\
$1: 10.1$ & $1: 8$
\end{tabular}

Ein ähnliches Verhältnis lälst sich auch bei vielen Doppelsalzen und metallischen Legierungen beobachten.

Z. anorg. Chem. XXX, 
wenden, deren lange prismatische Krystalle dem Zweige $B C$ (Figur 1) entsprechen, erhalten wir:

folglich

$$
m=2.37 ; n=1 \text {; }
$$

$$
2.37>x>1 \text {. }
$$

Dieser Ausdruck führt zu einer einfachsten Formel $\mathrm{Na}_{2} \mathrm{Tl}$ $(x=2)$. $\mathrm{Zu}$ diesem Typus gehört auch die Verbindung $\mathrm{Na}_{2} \mathrm{~Pb}$, die durch das hohe Temperaturmaximum $\left(420^{\circ}\right)$ unter den Legierungen des Bleis mit Natrium charakterisiert ist. Überhaupt wiederholt sich der Typus $\mathbf{M}_{2} \mathbf{R}$ sehr oft bei den Verbindungen der Metalle unter einander.

Viel weitcre Grenzen ergeben sich für die Substanz $\mathrm{Na}_{\mathrm{x}} \mathrm{Tl}$, die sich in der Form harter, gut ausgebildeter Oktaëder zwischen den Übergangspunkten $A$ und $B$ (Abschnitt $A B$ ) ausscheidet. Der Zusammensetzung der flüssigen Phasen nach haben wir:

$$
6.1>x>2.37 \text {. }
$$

Hieraus folgt, dals in der Formel $\mathrm{Na}_{\mathrm{x}} \mathrm{Tl}$ der dem Abschnitt $A B$ entsprechenden oktaëdrischen Krystalle der Koëffizient $x$ die einfachsten Werte von 3-6 haben kann. Die Substanz wird von der Luftfeuchtigkeit viel energischer als die beiden vorangehenden Körper NaTl und $\mathrm{Na}_{2} \mathrm{Tl}$ angegriffen.

Bei den obigen Ausführungen wurde stets angenommen, dafs die Zusammensetzung der festen Verbindungen immer bei allen Konzentrationsänderungen der flüssigen Phase konstant bleibt. In Wirklichkeit aber entspricht diese einfachste Annahme, besonders wo es sich um Verbindungen der Metalle mit einander handelt, nicht immer den Thatsachen; die Beobachtungen ergeben mehr oder minder bedeutende Abweichungen, und zwar sind dieselben verursacht 1) durch polymorphe Modifikationen, 2) durch feste Lösungen.

Der Kurvenzweig $B C$ zum Beispiel (Figur 1) könnte dem ersten Falle entsprechend nicht die Ausscheidung der neuen Verbindung $\mathrm{Na}_{\mathrm{x}} \mathrm{T}$, sondern eine zweite polymorphe Modifikation der Substanz $\mathrm{NaTl}$, welche bei niedrigen Temperaturen stabiler wäre als die dem Gebiete $C M D$ angehörende Modifikation, darstellen. Bei der geringen Differenz der den polymorphen Modifikationen eines und desselben Körpers angehörigen Lösungswärmen könnten jedoch die Änderungen der Winkelkoëffizienten der Schmelzpunktskurven (Lösungskurven) nicht so stark sein, wie wir es bei dem Schnittpunkte der beiden $Z$ weige $B C$ und $C M$ im Übergangspunkte $C$ finden. 
Im zweiten Falle könnte der Zweig $B C$, den wir wieder als Beispiel nehmen, verschiedene feste Lösungen des Natriumüberschusses in der chemischen Verbindung $\mathrm{Na}_{\mathbf{x}} \mathrm{Tl}$ darstellen. Das Gebiet homogener Zusammensetzung der festen und flüssigen Phase in diesem System ist begrenzt durch die Ordinate des Übergangspunktes $C$ einerseits und durch die des maximalen Schmelzpunktes der Verbindung NaTl andererseits. In dem durch den Kurvenzweig $B C$ umgrenzten Gebiete der festen Lösungen von $\mathrm{Na}_{x} \mathrm{Tl}$ mufs daher $x$ der obigen Ungleichheit (1)

$$
m>x>n
$$

genügen, in der $m=2.37 n=1$ und mithin $x$ wahrscheinlich $=2$ ist. So kann man aus der Lage des Übergangspunktes, auch wemu thatsächlich feste Lösungen vorliegen sollten, auf die wahrscheinliche Zusammensetzung der chemischen Verbindung (für den $\mathrm{Z}_{\text {weig }} B C$ bis $\mathrm{Na}_{2} \mathrm{Tl}$ ) schliefsen, die den Charakter der Krystallisation der festen Phase bedingt.

Komplizierter liegt der Fall, wenn ein Temperaturmaximum $A_{x} B$ (Figur 2) nicht mehr rationalen Atomverhältnissen der Komponenten $A$ und $B$ entspricht, wie wir es z. B. hei Blei-Thalliumlegierungen gefunden haben. ${ }^{1}$ Die Beziehungen derartiger irrationaler Maxima der Schmelzpunktskurven zu dem Gesetze der multiplen Proportionen, ebenso wie die Eigenschaften der entsprechenden festen Phasen bedürfen noch weiterer Untersuchungen.

Einheitlicher läfst sich das Diagramm der Legierungen des Kaliums mit Thallium - K $E N F \mathrm{Tl}$ (Figur 1) diskutieren. Hier sind die folgenden Punkte zu berücksichtigen: $E, N$ und $F$.

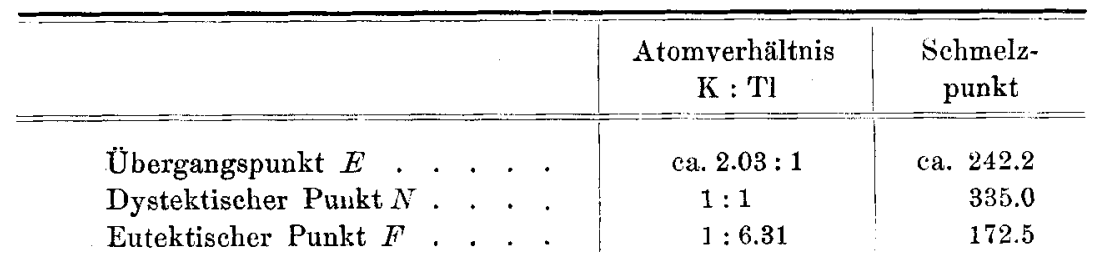

Die Schmelztemperatur des reinen Kaliums mufs etwas höher als $62.5^{\circ}$ liegen, sie lälst sich aber nur sehr ungenau bestimmen, da das unter einer Benzin-Vaselinschicht geschmolzene Metall beim Umrühren leicht in einzelne Kügelchen zerfällt, die sich nachher

1 Journ. russ. chem. Ges. 32 (1900), 830. 
auch beim Erkalten nicht wieder zu einer kompakten Masse vereinigen. Dieselbe Eigentümlichkeit erschwert auch die Gewinnung homogener Legierungen und man muss daher bei ihrer Darstellung möglichst das Umrühren vermeiden.

Beim Schmelzen erfährt das Kalium eine so starke Volumenzunahme, dafs es an die Oberfläche der deckenden Benzin-Vaselinschicht emporsteigt, falls diese nicht ein beträchtliches spezifisches Gewicht besitzt. Löst man jedoch die kleinste Menge Thallium im Kalium auf, so versinkt dieses sofort und bleibt, allmählich erstarrend, so lange auf dem Boden des Tiegels, bis die Temperatur auf $62.5^{\circ}$ gestiegen ist; dann zeigt das Metall wiederum die Tendenz, emporzusteigen. ${ }^{1}$ Dieses Verhältnis zeigt, dals der wohl die Krystallisation von freiem Kalium darstellende absteigende Kurvenzweig eine sehr geringe Ausdehnung hat, und dafs das Metall sich im eutektischen Punkte (bei $62.5^{\circ}$ ), der bei einer ganzen Reihe von Legierungen beobachtet wird, sich in reinem Zustande ausscheidet. Wahrscheinlich wird die Schmelztemperatur des Kaliums $\left(62.5^{\circ}\right)$ durch Zusatz ron Thallium erhöht.

Der Zweig $\mathrm{K} E$ auf der linken Seite des Diagramms gehört wohl der Verbindung $\mathrm{K}_{2} \mathrm{Tl}$ an; diese zersezt sich in dem wenig markierten Übergangspunkte $E$ unter Ausscheidung fester körniger Aggregate der Zusammensetzung KTl, denen ein hohes Schmelzpunktsmaximum zukommt (bei $335.0^{\circ}$ ). Die Verbindung KTl krystallisiert vorzüglich in Form von sprödẹn kompakten Würfeln, die langsam mit der Luftfeuchtigkeit reagieren. Bei der Krystallisation schwimmen die Krystalle KTl auf der Oberfläche der flüssigen Masse $(15-30 \% \mathrm{~K}$ ), woraus zu schliefsen ist, dafs diese Substanz, ebenso wie viele feste Alkaliamalgame (in denen Quecksilber überwiegt) ein spezifisches Gewicht besitzt, das kleiner als die entsprechende flüssige Lösung ist. Offenbar verliert der von manchen Forschern zur Bezeichnung der festen Phase bei Systemen ron inhomogenem Gleichgewicht angewandte Ausdruck "Bodenkörper" bei derartigen Substanzen seine Berechtigung.

Bekanntlich sind $\mathrm{K}$, Na und $\mathrm{Tl}$ im freien Zustande sehr weiche und plastische Körper; die Verbindungen dieser Alkalimetalle mit $\mathrm{Tl}$ sind dagegen viel härtere und sprödere Substanzen. ${ }^{2}$

${ }^{1}$ Diese Erscheinungen wurden von uns auch bei der Anflösung des Zinns im metallischen Natrium beobachtet.

${ }^{2}$ Nach den Untersuchungen von K. L. WINKLER (Joum. prakt. Chem. 102 (1867), 279 steht aufser Zweifel, dafs das nächste Analogon des Thalliums 
Auf Grund aller dieser Beobachtungen dürfte wohl erwiesen sein, dafs das Thallium, gleich seinen Analogen in der 11. horizontalen Reihe des periodischen Systems - $\mathrm{Hg}$, $\mathrm{Pb}, \mathrm{Bi}$ - eine Reihe von Verbindungen mit Alkalimetallen bildet, in denen die Eigenschaften der Komponenten wesentliche Änderung erleiden.

Wenn wir die typischen Verbindungen, die den Schmelzpunktsmaxima dieser Metallverbindungen entsprechen, mit einander vergleichen, so erhalten wir folgende Reihe, deren einzelne Glieder sich z. B. bei den Natriumverbindungen regelmälsig in der Zusammensetzung ändern:

$$
\mathrm{NaHg}{ }_{2}, \mathrm{NaTl}, \mathrm{Na}_{2} \mathrm{~Pb}, \mathrm{Na}_{3} \mathrm{Bi}
$$

Es findet also mit steigendem Atomgewicht und mit Zunahme der sauren Eigenschaften des Schwermetalles ein gesetzmäfsiges Anwachsen der Anzahl der Alkaliatome auf ein Atom des Schwermetalles statt.

\section{Legierung des Thalliums mit Cadmium, Zinn und Quecksilber.}

Die Untersuchungsmethode dieser Systeme war dieselbe, wie bei den vorigen Legierungen. Die ermittelten Resultate sind in den Tabellen III, IV, V (Seite 102-105) angeführt und in der Figur 3 graphisch dargestellt.

(S. Tabelle III-V, S. 102-105.)

Die Diagramme der 'Thalliumlegierungen mit Sn, Cd entsprechen dem einfachen Typus der Schmelzpunktskurven und bestehen aus zwei, die Ausscheidung der einzelnen Metalle darstellenden Linien, die sich in den eutektischen Punkten $F$ und $E$ (Figur 3) schneiden. Die Lage der letzteren wird durch folgende Angaben bestimmt:

\begin{tabular}{|c|c|c|}
\hline Eutektische Punkte & Atomverhältnis & Schmelzpunkt \\
\hline$E$ (Legierungen $\mathrm{Sn}$ mit $\mathrm{Tl}$ ) & $\mathrm{Sn}: \mathrm{TI}=2.2: 1$ & $170.2^{\circ}$ \\
\hline$F$ (Legierungen $\mathrm{Cd}$ mit $\mathrm{T}$ ) & $\mathrm{Cd}: \mathrm{Tl}=1: 267$ & $203.5^{\circ}$ \\
\hline
\end{tabular}

in der III. Gruppe des periodischen Systems - das Indium auch chemische Verbindungen mit Natrium bildet. Bei der Darstellung mittels des metallischen Natriums aus dem Oxyd giebt das Indium eine spröde Legierung, aus der sich das Natrium durch Wasser nicht vollständig entfernen lärst. 
Tabelle III.

Legierungen des Thalliums mit Zinn.

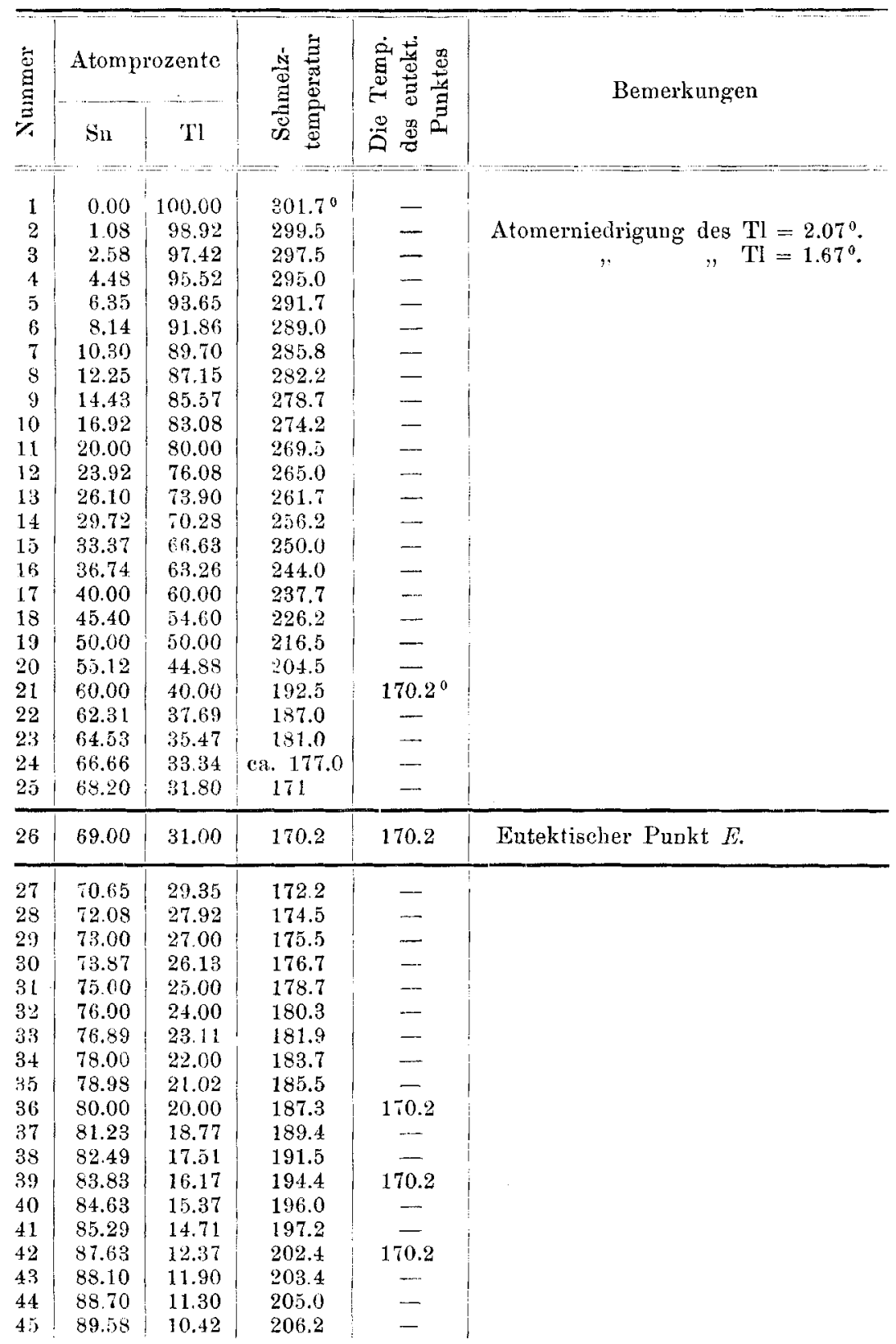


Tabelle III (Fortsetzung).

\begin{tabular}{|c|c|c|c|c|c|}
\hline \multirow{2}{*}{ 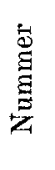 } & \multicolumn{2}{|c|}{ Atomprozente } & \multirow{2}{*}{ 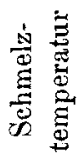 } & \multirow{2}{*}{ 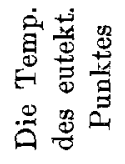 } & \multirow{2}{*}{ Bemerkungen } \\
\hline & Sn & $\mathrm{Tl}$ & & & \\
\hline 46 & 90.71 & 9.29 & 208.6 & - & \\
\hline 47 & 91.85 & 8.15 & 211.2 & — & \\
\hline 48 & 93.03 & 6.97 & 213.7 & - & \\
\hline 49 & 94.49 & 5.51 & 217.0 & - & \\
\hline 50 & 96.17 & 3.83 & 221.1 & $\ldots$ & Atomerniedrigung des $\mathrm{Zinns}=2.82^{\circ}$ \\
\hline 51 & 97.96 & 2.04 & $220 \overline{0} .7$ & - & $\|\quad\| \quad=2.90^{\circ}$ \\
\hline 52 & 100.00 & 0.00 & 231.5 & - & \\
\hline
\end{tabular}

Tabelle IV.

Legierungen des Thalliums mit Cadminm.

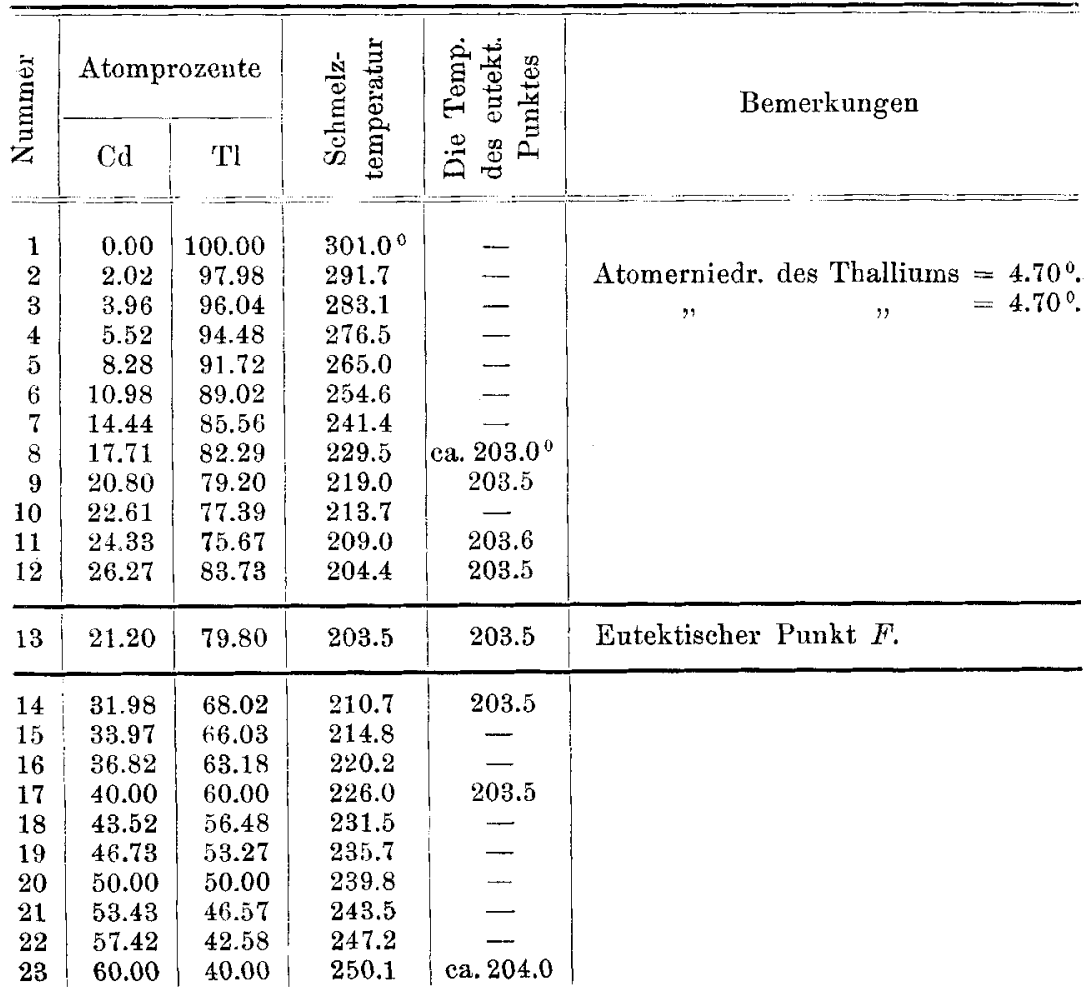


Tabelle IV (Fortsetzung).

\begin{tabular}{|c|c|c|c|c|c|}
\hline 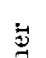 & \multicolumn{2}{|c|}{ Atomprozente } & \multirow{2}{*}{ 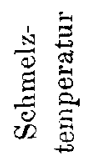 } & \multirow{2}{*}{ 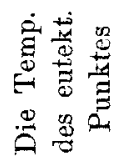 } & \multirow{2}{*}{ Bemerkungen } \\
\hline & $\mathrm{Cd}$ & $\mathrm{Tl}$ & & & \\
\hline 24 & 62.43 & 37.57 & $252.4^{\circ}$ & $\ldots$ & \\
\hline 25 & 65.2 & 34.75 & 254.4 & - & \\
\hline 26 & 66.67 & 83.33 & 256.0 & - & \\
\hline 27 & 68.97 & 31.03 & 257.8 & - & \\
\hline 28 & 70.37 & 29.63 & 259.15 & - & \\
\hline 29 & 72.20 & 27.80 & 260.7 & - & \\
\hline 30 & 74.22 & 25.78 & 263.0 & - & \\
\hline 31 & 75.07 & 24.93 & 263.8 & - & \\
\hline 32 & 77.25 & 22.75 & 266.5 & $\ldots$ & \\
\hline 33 & 79.25 & 20.75 & 268.5 & $203.7^{\circ}$ & \\
\hline 34 & 81.09 & 18.91 & 271.2 & - & \\
\hline 35 & 82.49 & 17.51 & 273.0 & - & \\
\hline 36 & 84.33 & 15.67 & 275.8 & 204.0 & \\
\hline 37 & 85.82 & 14.18 & 278.3 & - & \\
\hline 38 & 91.06 & 8.94 & 290.2 & - & \\
\hline 39 & 92.53 & 7.47 & 293.6 & - & \\
\hline 40 & 94.01 & 5.99 & 297.7 & - & \\
\hline 41 & 95.71 & 4.29 & 302.7 & - & \\
\hline 42 & 97.54 & 2.46 & 310.0 & - & Atomerniedr. des Cadmiums $=4.58^{\circ}$. \\
\hline 43 & 99.05 & 0.95 & 316.5 & - & $=4.78^{\circ}$. \\
\hline 44 & 100.00 & 0.00 & 321.0 & - & \\
\hline
\end{tabular}

Tabelle V.

Tegierungen des Thalliums mit Quecksilber.

\begin{tabular}{|c|c|c|c|c|c|}
\hline$\dot{\overrightarrow{3}}$ & \multicolumn{2}{|c|}{ Atomprozente } & $\dot{N}$ & $\dot{\dot{\theta}}+\vec{J}$ & \multirow{2}{*}{ Bemerkuugen } \\
\hline $\bar{Z}$ & $\mathrm{Hg}$ & 'Tl & $\tilde{\Xi}$ & $\stackrel{9}{0}$ & \\
\hline & & & $==$ & $==$ & \multirow{15}{*}{$\begin{aligned} \text { Atomemiedrigung des } \mathrm{Tl} & =4.22^{\circ} . \\
$\[ , \quad, \quad \]$ & =4.23^{\circ} .\end{aligned}$} \\
\hline 1 & 0.00 & 100.00 & $301.7^{\circ}$ & $\sim$ & \\
\hline 2 & 1.44 & 98.56 & $295 . \overline{7}$ & - & \\
\hline 3 & 2.72 & 97.28 & 290.5 & - & \\
\hline 4 & 4.89 & 95.11 & 281.5 & - & \\
\hline 5 & 7.07 & 92.93 & 273.0 & - & \\
\hline 6 & 9.19 & 90.81 & 263.7 & - & \\
\hline 7 & 11.30 & 88.70 & 255.5 & - & \\
\hline 8 & 13.50 & 86.50 & 246.0 & - & \\
\hline 9 & 16.56 & 83.44 & 233.4 & - & \\
\hline 10 & 19.34 & 80.66 & 221.0 & - & \\
\hline 11 & 22.73 & 77.27 & 206.5 & - & \\
\hline 12 & 25.07 & 74.93 & 196.1 & - & \\
\hline 13 & 29.00 & 71.00 & 180.0 & -- & \\
\hline 14 & 33.42 & 66.58 & 156.7 & -- & \\
\hline
\end{tabular}


Tabelle V (Fortsetzung).

\begin{tabular}{|c|c|c|c|c|c|}
\hline \multirow{2}{*}{$\begin{array}{l}\stackrel{\Xi}{\Xi} \\
\stackrel{\Xi}{\Xi} \\
\text { Z }\end{array}$} & \multicolumn{2}{|c|}{ Atomprozente } & \multirow{2}{*}{ 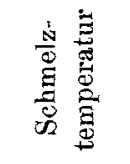 } & \multirow{2}{*}{ 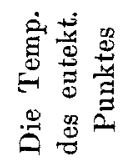 } & \multirow{2}{*}{ Bemerkungen } \\
\hline & $\mathrm{Hg}$ & 'TI & & & \\
\hline $\begin{array}{l}15 \\
16 \\
17 \\
18 \\
19 \\
\end{array}$ & $\begin{array}{l}36.77 \\
40.20 \\
45.36 \\
50.06 \\
57.90 \\
\end{array}$ & $\begin{array}{l}63.23 \\
59.80 \\
54.64 \\
49.94 \\
42.10 \\
\end{array}$ & $\begin{array}{r}138.5 \\
121.0 \\
93.0 \\
\text { ca. } 67.0 \\
\text { ca. } 20.0 \\
\end{array}$ & $\begin{array}{l}- \\
\overline{-} \\
3.5^{\circ} \\
3.5\end{array}$ & \\
\hline 20 & 60.00 & 40.00 & 3.5 & - & Eutektischer Punkt $C$. \\
\hline $\begin{array}{l}21 \\
22\end{array}$ & $\begin{array}{l}62.18 \\
64.54\end{array}$ & $\begin{array}{l}37.82 \\
35.46\end{array}$ & $\begin{array}{r}9.0 \\
12.8\end{array}$ & - & \\
\hline 23 & 66.67 & 33.33 & 15.0 & - & $\begin{array}{l}\text { Dystek tischer Punkt } B ; \text { THg }_{2} \text { ent- } \\
\text { bält } 66.67 \% \mathrm{Hg} \text { und } 33.33 \% \text { T1 }\end{array}$ \\
\hline $\begin{array}{l}24 \\
25 \\
26 \\
27 \\
28 \\
29 \\
30 \\
\end{array}$ & $\begin{array}{l}68.00 \\
69.72 \\
72.10 \\
75.00 \\
77.70 \\
80.00 \\
84.12 \\
\end{array}$ & $\begin{array}{l}32.00 \\
30.28 \\
27.90 \\
25.00 \\
22.30 \\
20.00 \\
15.88 \\
\end{array}$ & $\begin{array}{r}15.0 \\
14.5 \\
12.0 \\
5.5 \\
-3.0 \\
-11.5 \\
\text { ca. }-30.0 \\
\end{array}$ & $\begin{array}{c}- \\
\overline{-} \\
\overline{-} \\
-60.0 \\
-60.0 \\
\end{array}$ & \\
\hline 31 & 91.66 & 8.34 & -60.0 & - & Eutektischer Punkt $A$. \\
\hline $\begin{array}{l}32 \\
33 \\
84 \\
35 \\
36\end{array}$ & $\begin{array}{r}93.22 \\
94.32 \\
96.89 \\
98.76 \\
100.00\end{array}$ & $\begin{array}{l}6.78 \\
5.68 \\
3.11 \\
1.24 \\
0.00\end{array}$ & $\begin{array}{c}-56.0 \\
-53.0 \\
-46.0 \\
\text { ca. }-41.0 \\
-39.0\end{array}$ & $\begin{array}{l}- \\
z \\
=\end{array}$ & $\begin{array}{cc}\text { Atomerniedrigung } & -2.32^{\circ} \\
, " & -1.63^{\circ}\end{array}$ \\
\hline
\end{tabular}

(S. Figur 3, S. 106.)

Der allgemeinen Form der Kurven nach stehen diese Thalliumlegierungen den entsprechenden Bleilegierungen sehr nahe. Der für Linie CdF (zwischen $30-40 \%$ ) charakteristische Knickpunkt wiederholt sich z. B. auch in dem $\mathrm{CdPb}$-Diagramm. ${ }^{1}$ Dasselbe gilt auch für die hohe Atomdepression, die sich bei den Legierungen zeigt, wie es aus dem Vergleiche der bei den geringen Konzentrationen des aufgelösten Metalles berechneten Erniedrigungsgröfsen ersichtlich ist (siehe Tabelle VI und VII).

${ }^{1}$ Die Untersuchung der Schmelzbarkeit des Systems $\mathrm{Pb}+\mathrm{Cd}$ wurde im Chem. Laboratorium des Berginstitutes zuerst von A. N. Kosnezofp ausgeführt, dann fortgesetzt von A. KAPP (siehe A. KAPP, Über vollständige Gefrierpunktskurven binärer Legierungen. Inang.-Dissertation, Königsberg 1901). 
Die starke Verminderung der Atomdepression des Thalliums und des Bleis bei der Auflösung von Cadmium und Zinn hängt

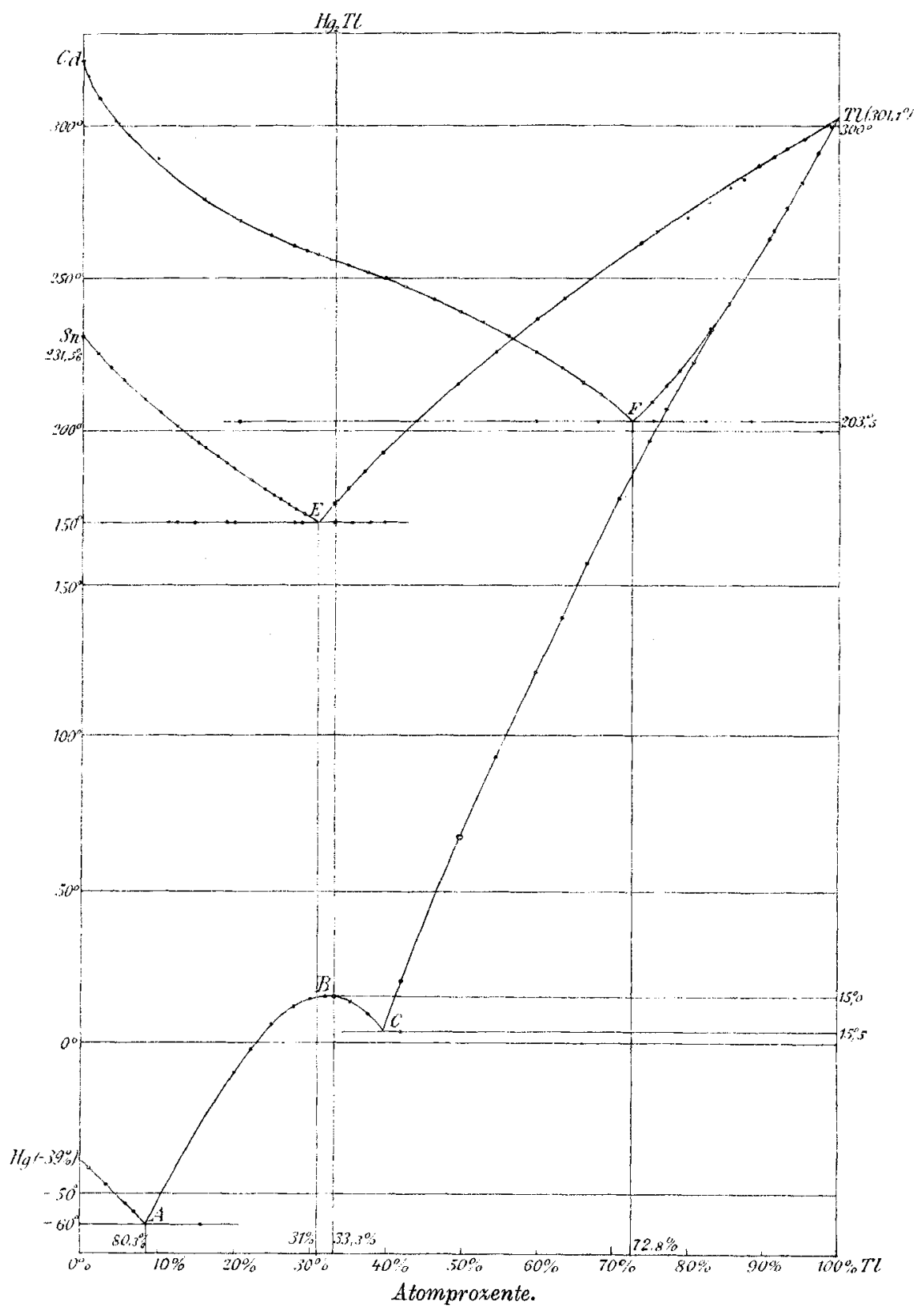

Fig. 3. Legierung des Thalliums mit Cadmium, Zinn und Quecksilber. 
höchstwahrscheinlich von der Ausscheidung fester Lösungen ab. In Übereinstimmung damit steht auch der Umstand, dals die Härte der Legierungen Sn und TI bedeutend grölser ist, als die jeder einzelnen Komponente.

Tabelle VI.

\begin{tabular}{|c|c|c|}
\hline \multirow[b]{2}{*}{ Aufgelöstes Metall } & \multicolumn{2}{|c|}{ Lösungsmittel: } \\
\hline & $\begin{array}{c}\text { Tl, theoretische } \\
\text { Atomerniedigrung } \\
=\text { ca. } 6.0\end{array}$ & $\begin{array}{l}\text { Blei, theoretische } \\
\text { Atomerniedrigung } \\
=\text { ca. } 6.5\end{array}$ \\
\hline Zinn & $2.0-1.7$ & 1.7 \\
\hline $\mathrm{Cd}$. & $2.0-4.7$ & 4.1 \\
\hline
\end{tabular}

Tabelle VII.

\begin{tabular}{|c|c|c|}
\hline \multirow[b]{2}{*}{ Aufgelöstes Metall } & \multicolumn{2}{|c|}{ Lösungsmittel: } \\
\hline & $\begin{array}{c}\text { Zinn, theoretische } \\
\text { Atomerniedrigung } \\
=3.0\end{array}$ & $\begin{array}{c}\mathrm{Cd} \text {, theoretische } \\
\text { Atomerniedrigung } \\
=4.5\end{array}$ \\
\hline $\mathrm{Tl}$ & 2.9 & 4.5 \\
\hline Blei. . & 2.8 & 4.4 \\
\hline
\end{tabular}

Für ein dem vorigen nahe stehendes System, nämlich für Legierungen des Zinus mit Wismut, in denen das Zinn auch eine verhältnismälsig kleine Atomerniedrigung besitzt (2.4 statt 3.0) äufsert sich, unter dem Mikroskop betrachtet, die Bildung der festen Lösungen in F'orm der sogenannten sekundären Struktur der Zinnkrystalle.

Das Schrnelzdiagramm der Thalliumamalgame unterscheidet sich scharf von den eben beschriebenen durch die Existenz eines, zwischen den eutektischen Punkten $C$ und $A$ auftretenden Maximums $B$ der Schmelztemperatur $15^{\circ}$ mit 33.33 Atomprozenten Tl. Der Zweig $A B C$ gehört der chemischen Verbindung $\mathrm{THHg}_{2}$, die ohne Zersetzung schmilzt und nach dem Typus der Alkaliamalgame $\mathrm{NaHg}_{2}$ und $\mathrm{KHg}_{2}$ zusammengesetzt ist; diese haben bekanntlich hohe Temperaturmaxima. Die Existenz der Verbindung $\mathrm{TlHg}_{2}$ lälst darauf schliefsen, dafs das Thallium dem Quecksilber gegenüber die Funktion des Alkalimetalles ausübt.

Rechts rom eutektischen Punkte $C$ (bei $3.5^{\circ} ; 40.0$ Atomprozente Tl) steigt die Schmelzpunktskurve ununterbrochen bis $301.7^{\circ}$ und entspricht den flüssigen Amalgamen, die im Gleichgewicht mit den Krystallen des metallischen Natriums, oder richtiger, mit dessen 
festen Lösungen mit Quecksilber sich befinden. Hiermit steht auch die verhältnismälsig geringe Atomdepression des Thalliums (4.2 statt $6.5-6.8$ ) in Übereinstimmung.

Bei niederen Temperaturen löst sich das Thallium in grolsen Mengen im Quecksilber auf und bewirkt eine allmähliche Erniedrigung des Schmelzpunktes (Zweig $\mathrm{Hg} A$ ). Der untere eutektische Punkt $A$, in welchem eine totale Erstarrung der Amalgame erfolgt, befindet sich bei -60 und entspricht einem Prozentgehalt von 8.34 Atomprozenten des Tl. Diese Legierung hat die niedrigste Erstarrungstemperatur, die bisher bei metallischen Substanzen beobachtet ist. Die oben angeführte Analogie des Thalliums und der Alkalimetalle beschränkt sich nicht nur auf die Verbindungen des Thalliums mit dem Quecksilber, sondern kann auch in dessen Legierungen mit Blei und Wismut verfolgt werden; diesbezügliche Versuche bilden den Gegenstand unserer weiteren Untersuchungen.

St. Pelersburg, Chemisches Laboratorium des elektrotechnischen Instituts.

Bei der Redaktion eingegangen am 26. November 1901. 